Regards sur l'économie allemande

Bulletin économique du CIRAC

118-119| 2015

Varia

\title{
Réforme du droit des successions allemand applicable aux entreprises
}

François Hellio et Annett Kenk

\section{(2) OpenEdition}

\section{Journals}

Édition électronique

URL : http://journals.openedition.org/rea/4884

DOI : $10.4000 /$ rea.4884

ISSN : 1965-0787

Éditeur

CIRAC

Édition imprimée

Date de publication : 31 décembre 2015

Pagination : 5-13

ISSN : 1156-8992

Distribution électronique Cairn

\section{CAIRN, INFO}

CHERCHER, REPÉRER, AVANCER.

\section{Référence électronique}

François Hellio et Annett Kenk, « Réforme du droit des successions allemand applicable aux entreprises », Regards sur l'économie allemande [En ligne], 118-119 | décembre 2015, mis en ligne le 31 décembre 2017, consulté le 06 mai 2019. URL : http://journals.openedition.org/rea/4884 ; DOI : $10.4000 /$ rea.4884 


\section{Réforme du droit des successions allemand applicable aux entreprises}

\section{François Hellio, Annett Kenk}

L'entreprise - et tout particulièrement la familiale - est au cœur du modèle économique allemand. Dès lors, le traitement fiscal de la transmission d'entre prise est un sujet central, et la réforme en cours du régime des successions donne lieu à d'ardentes négociations.

Depuis la suppression de l'impôt sur la fortune en 1997, le capital n'est taxé en Allemagne qu'au titre de sa transmission à titre gratuit. La transmission d'entreprise ne fait en principe pas exception, même si le droit allemand prévoit depuis longtemps la possibilité d'effectuer ce type de transmissions dans des conditions privilégiées, pouvant aller jusqu'à une exonération. La situation française est a priori très différente puisque l'impôt sur la fortune, instauré en 1981, existe toujours (après une brève abrogation entre 1986 et 1988). La loi prévoit toutefois un principe d'exonération totale pour les biens professionnels, et, pour les membres d'un groupe familial qui ne pourraient pas tous bénéficier de cette exonération totale, la possibilité de bénéficier d'une exonération à $75 \%$ par la conclusion d'un pacte (Dutreil) par lequel des engagements (collectifs puis individuels) de détention des titres de la société concernée sont pris. En matière de droits de mutation à titre gratuit (droits de donation ou de succession), le principe du pacte (Dutreil) permet également une exonération à $75 \%$ (étant précisé que des donations en démembrement de propriété peuvent, en pratique, encore réduire l'assiette des droits exigibles).

Finalement, la loi française apparaît globalement plus simple que la loi allemande en matière de transmission du patrimoine professionnel dans un contexte familial. Il faut toutefois souligner que l'absence de clarification de l'administration fiscale sur la définition de la holding animatrice est actuellement la source d'une regrettable insécurité juridique, tant en matière d'impôt sur la fortune que de droits de mutation à titre gratuit.

Mais, en la matière, l'insécurité juridique apparaît encore plus marquée en Allemagne. En effet, les développements récents sur la constitutionnalité du dispositif allemand en vigueur ont contraint le législateur à modifier son système d'imposition en vue de le rendre conforme à la Loi fondamentale, sans toutefois trop perdre de sa souplesse. Cet exercice, non encore totalement abouti, révèle toute la complexité liée aux différentes exigences posées par le Juge Constitutionnel. Les débats sur le projet de loi en cours visant à réformer le traitement fiscal de la transmission d'entreprises en Allemagne sont rendus d'autant plus complexes qu'ils semblent clairement prendre un tour politique, le Bundesrat s'opposant au projet de loi proposé par le gouvernement fédéral. Le délai jusqu'au 30 juin 2016 posé par Karlsruhe semble à ce jour difficilement tenable.

\section{Assujettissement à l'impôt en France et en Allemagne}

En Allemagne, les successions et donations sont assujetties à l'impôt lorsque le défunt, le donateur ou l'héritier, le donataire, a son lieu de séjour habituel ou sa résidence en Allemagne. De plus, un citoyen allemand est assujetti à l'impôt de façon illimitée lorsqu'il séjourne moins de cinq ans à l'étranger ou lorsqu'il est employé par une personne morale de droit public et perçoit un salaire d'une

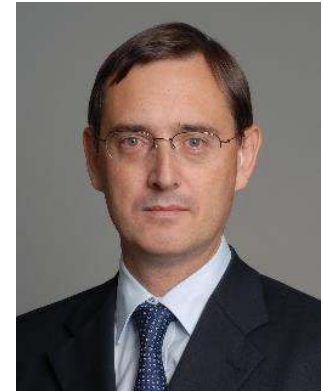

François Hellio, avocat associé au sein du cabinet CMS Bureau Francis Lefebvre

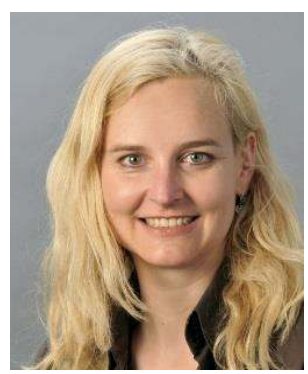

Annett Kenk, avocate et conseiller fiscal au sein du cabinet CMS Hasche Sigle

Allemagne : assujettissement illimité 
Donation/succession : assujettissement limité

RFA/France convention d'élimination des doubles impositions

Classes d'imposition en Allemagne

Ordres d'imposition en France caisse publique nationale. Dans les cas d'un assujettissement illimité (uneingeschränkte Steuerpflicht) à l'impôt allemand, tous les biens sont imposables en Allemagne.

La donation ou la succession peut faire l'objet d'un assujettissement limité (eingeschränkte Steuerpflicht) lorsque certains biens qui ont un rapport avec le territoire allemand sont transmis. C'est le cas par exemple des :

- immeubles ou établissements stables situés en Allemagne,

- participations dans une société de capitaux qui a son siège ou sa direction sur le territoire national, lorsqu'au moins $10 \%$ du capital social y sont détenus,

- créances issues de prêts participatifs ou

- associés d'une société type tacite (Stille Gesellschaft). II s'agit d'un mode de financement spécifique au droit allemand, par lequel un investisseur ne souhaite ni être connu en tant que tel de l'extérieur, ni participer activement à la gestion de l'activité, et entend obtenir une partie du profit réalisé par l'entreprise en contrepartie de sa participation.

L'Allemagne et la France ont conclu le 12 octobre 2006 une convention d'élimination des doubles impositions en matière de donations et successions. Cette convention est applicable lorsque le défunt ou donateur, au moment du décès ou de la donation, avait sa résidence dans l'un des Etats contractants ou dans les deux Etats. La succession ou la donation est en principe imposée de façon illimitée dans l'Etat où le défunt ou donateur a sa résidence fiscale. II existe cependant des exceptions s'agissant des actifs d'une entreprise et des biens immobiliers qui sont à imposer dans l'Etat du lieu de situation. Puisqu'en vertu de la convention, la France et l'Allemagne prévoient un système d'imputation afin d'éliminer les doubles impositions, l'imposition est toujours fixée au plus haut des deux niveaux d'imposition établis respectivement dans chaque Etat.

\section{Classes d'imposition, abattements, taux d'imposition}

La loi allemande différencie trois classes d'imposition qui sont fonction des liens de parenté - comme en droit français - existant avec le défunt ou le donateur. Ces classes sont également déterminantes pour les abattements et les taux d'imposition applicables.

\begin{tabular}{|c|c|c|}
\hline Classe I & $\begin{array}{l}\dot{\bullet} \\
\dot{ } \\
\bullet\end{array}$ & $\begin{array}{l}\text { Conjoint et partenaire enregistré } \\
\text { Enfants et enfants du conjoint } \\
\text { Petits-enfants } \\
\text { Parents et grands-parents (en cas d'héritage) }\end{array}$ \\
\hline Classe II & $\begin{array}{l}\bullet \\
\bullet \\
\bullet \\
- \\
- \\
-\end{array}$ & $\begin{array}{l}\text { Parents et grands-parents (en cas de donation) } \\
\text { Frères et sœurs } \\
\text { Neveux et nièces } \\
\text { Beaux-parents } \\
\text { Belles-filles/beaux-fils et beaux-parents } \\
\text { Conjoint divorcé et ex-partenaire enregistré }\end{array}$ \\
\hline Classe III & • & Toutes autres personnes \\
\hline
\end{tabular}

En France, on distingue, par rang de priorité, quatre ordres établis hiérarchiquement.

\begin{tabular}{|l|ll|}
\hline Ordre I & $\bullet$ & Descendants : enfants, petits-enfants,... \\
\hline Ordre II & $\bullet$ & $\begin{array}{l}\text { Ascendants privilégiés : père, mère } \\
\text { Collatéraux privilégiés : frères et sœurs et leurs descendants } \\
\text { jusq'au 6ème degré }\end{array}$ \\
\hline Ordre III & $\bullet$ & Ascendants ordinaires : grands-parents, arrière-grands-parents... \\
\hline Ordre IV & $\bullet$ & $\begin{array}{l}\text { Collatéraux ordinaires : oncles, tantes, grands-oncles, grands- } \\
\text { tantes, cousins, cousines jusqu'au 6 }\end{array}$ \\
\hline
\end{tabular}




\begin{tabular}{|l|l|c|}
\hline Classe & Acquéreur & Abattement en $€$ \\
\hline I. & Conjoint et partenaire enregistré & $\mathbf{5 0 0 0 0 0}$ \\
\hline I. & Enfants et enfants du conjoint & $\mathbf{4 0 0 0 0 0}$ \\
\hline I. & Petits-enfants & $\mathbf{2 0 0 0 0 0}$ \\
\hline I. & Parents et grands-parents (en cas d'héritage) & $\mathbf{1 0 0 0 0 0}$ \\
\hline II. & $\begin{array}{l}\text { Parents et grands-parents (en cas de donation), sœurs et } \\
\text { frères etc. }\end{array}$ & $\mathbf{2 0 0 0 0}$ \\
\hline III. & Toutes autres personnes & $\mathbf{2 0 0 0 0}$ \\
\hline
\end{tabular}

Abattements.

En Allemagne...

... en France

\begin{tabular}{|l|c|}
\hline Acquéreur & Abattement en $€$ \\
\hline Ascendants et enfants en ligne directe & 100000 \\
\hline Frères et sœurs & 15932 \\
\hline Neveux et nièces & 7967 \\
\hline Toutes autres personnes (pas en matière de donation) & 1594 \\
\hline Handicapés & 159325 \\
\hline
\end{tabular}

Abattements particuliers supplémentaires en matière de droits de donations

\begin{tabular}{|l|c|}
\hline Acquéreur & Abattement en $€$ \\
\hline Epoux et partenaires d'un Pacs & 80724 \\
\hline Petits-enfants & 31865 \\
\hline Arrière-petits-enfants & 5310 \\
\hline
\end{tabular}

\begin{tabular}{|c|c|c|c|}
\hline \multicolumn{3}{|c|}{ Taux } \\
\hline $\begin{array}{l}\text { Valeur d'acquisition } \\
\text { jusqu'à } \ldots \in \text { inclus }\end{array}$ & \multicolumn{3}{c|}{ Classe } \\
\cline { 2 - 4 } & I & II & III \\
\hline $\mathbf{7 5 0 0 0}$ & $7 \%$ & $15 \%$ & $30 \%$ \\
\hline $\mathbf{3 0 0 0 0 0}$ & $11 \%$ & $20 \%$ & $30 \%$ \\
\hline $\mathbf{6 0 0 0 0 0} 000000$ & $15 \%$ & $25 \%$ & $30 \%$ \\
\hline $\mathbf{1 3 0 0 0 0 0 0}$ & $19 \%$ & $30 \%$ & $50 \%$ \\
\hline $\mathbf{2 6 0 0 0 0 0 0}$ & $23 \%$ & $35 \%$ & $50 \%$ \\
\hline plus & $27 \%$ & $40 \%$ & $50 \%$ \\
\hline
\end{tabular}

\begin{tabular}{|c|c|c|c|}
\hline $\begin{array}{l}\text { Valeur } \\
\text { d'acquisition } \\
\text { jusqu'à ... } € \text { inclus }\end{array}$ & $\begin{array}{l}\text { Ligne directe (succession) } \\
\text { Epoux et partenaires d'un pacs } \\
\text { (donation) }\end{array}$ & $\begin{array}{c}\text { Parents } \\
\text { jusqu'au } 4^{\text {ème }} \\
\text { degré }\end{array}$ & $\begin{array}{l}\text { Parents au-delà } \\
\text { du } 4^{\text {ème }} \text { degré }\end{array}$ \\
\hline 8072 & $5 \%$ & \multirow{7}{*}{$55 \%$} & \multirow{7}{*}{$60 \%$} \\
\hline 12109 & $10 \%$ & & \\
\hline 15932 & $15 \%$ & & \\
\hline 552324 & $20 \%$ & & \\
\hline 902838 & $30 \%$ & & \\
\hline 1805677 & $40 \%$ & & \\
\hline supérieure & $45 \%$ & & \\
\hline
\end{tabular}

... en France 


\begin{tabular}{|c|c|}
\hline Valeur d'acquisition jusqu'à ... $€$ inclus & Frères et sœurs \\
\hline $\mathbf{2 4} \mathbf{4 3 0}$ & $35 \%$ \\
\hline supérieure & $45 \%$ \\
\hline
\end{tabular}

\section{Le traitement fiscal privilégié de la transmission d'entreprise en Allemagne}

Au vu de la multitude d'entreprises familiales (environ $95 \%$ du total des entreprises), qui constituent le fondement du système économique allemand, le législateur a largement établi des règles d'exonération d'impôt pour les entreprises.

\section{L'actuelle règle de faveur en vigueur jusqu'au 30 juin 2016}

Deux types d'exonération...

... sous condition

Maintien de la masse salariale
Conformément aux $\S 13$ a et suivants de la Loi sur les droits de successions (Erbschaftsteuergesetz), les actifs d'une entreprise ou les biens agricoles ou forestiers ne sont pas imposés sous réserve des conditions y mentionnées, et ce à hauteur de $85 \%$ des actifs de l'entreprise (exonération légale, Regelverschonung) ou sur demande, à hauteur de $100 \%$ (exonération sur option, Optionsverschonung).

Les biens faisant l'objet du régime de faveur selon l'actuelle législation sont :

- les biens agricoles et forestiers,

- les actifs d'une entreprise: l'acquisition d'une entreprise entière, d'une branche d'activité ou des parts d'une société de personnes,

- une détention substantielle des actions d'une société de capitaux correspondant à une participation $>25 \%$.

En principe, les actifs d'une entreprise ne bénéficient de ces règles d'exonération que lorsque celle-ci ne détient pas plus de $50 \%$ de patrimoine administratif. Le patrimoine administratif susceptible d'ôter le bénéfice de toute exonération est composé :

- des participations ouvrant droit à la jouissance de terrains mis à disposition, de parcelles de terrains, ou à tout autre droit immobilier équivalent,

- des actions dans des sociétés de capitaux correspondant à une participation $<25 \%$, des valeurs mobilières et créances comparables,

- des œuvres d'art,

- des moyens financiers tels que des avoirs financiers ou commerciaux,

- des participations dans des sociétés de personnes et des actions substantielles dans des sociétés de capitaux disposant de plus de $50 \%$ de patrimoine administratif, ainsi que du patrimoine administratif « jeune » qui a été apporté à l'actif de l'entreprise au cours des deux dernières années.

En France, une telle limitation du champ de l'exonération relative à un patrimoine administratif n'existe pas.

En Allemagne, une autre condition pour obtenir le bénéfice de l'exonération réside dans le maintien de la masse salariale pendant plus de cinq ans dans le cadre de l'exonération légale, et pendant plus de sept ans dans le cadre de l'exonération sur option. Cette condition n'existe nullement en droit français.

C'est la moyenne de la masse salariale pendant les cinq derniers exercices qui est déterminante. Elle comprend toutes les rémunérations payées aux salariés au cours de l'exercice. Dans la masse salariale, il faut entendre tous les salariés à proportion d'une participation de plus de $25 \%$ dans la société. La même règle s'applique pour les participations dans des sociétés dont le siège se situe dans l'UE ou l'EEE.

Dans le cadre de l'exonération légale, l'entrepreneur doit conserver la masse salariale à hauteur de $400 \%$ sur une période de cinq ans; dans le cadre de l'exonération sur option, la masse salariale doit être conservée à hauteur de 
$700 \%$ (au lieu de $400 \%$ ) sur une période de sept ans (au lieu de cinq ans). Si les exigences relatives à la masse salariale ne sont pas remplies, l'exonération n'est alors rétroactivement plus permise, à la proportion de la masse salariale effective et de la masse salariale en cause. La règle relative à la masse salariale ne trouve à s'appliquer que pour les entreprises ayant plus de 20 salariés. Ainsi, les entrepreneurs qui acquièrent, par voie de succession ou de donation, une entreprise qui compte moins de 20 salariés peuvent obtenir le bénéfice de l'exonération partielle de droits afférente à la transmission sans avoir aucune contrainte liée au maintien d'une certaine masse salariale.

En outre, une condition supplémentaire est que l'acquéreur conserve l'entreprise cinq ans s'agissant de l'exonération légale, et sept ans dans le cas de l'exonération sur option. En France, ce délai de conservation est de quatre ans.

\section{La décision de la Cour de Karlsruhe du 17 décembre 2014}

Le Tribunal constitutionnel fédéral devait se prononcer sur la constitutionnalité de l'exonération légale. Dans son principe, la Cour adhère au régime de faveur des entreprises qui est selon elle justifié, sous réserve que le législateur en améliore certaines caractéristiques.

Plus précisément, la Cour constitutionnelle a critiqué les points suivants :

L'exonération d'impôt accordée pour les actifs d'une entreprise est disproportionnée dans la mesure où elle s'applique au-delà des seules petites et moyennes entreprises sans aucun examen des besoins réels des entreprises concernées. La Cour rappelle que les grosses entreprises ne sont pas la véritable cible du dispositif.

A l'inverse, la règle relative à la masse salariale privilégie les entreprises ayant jusqu'à 20 salariés également de façon disproportionnée. Ainsi, plus de $90 \%$ des entreprises sont dispensées d'impôt, et l'argument pivot pour le privilège accordé - à savoir le maintien ou la création d'emplois - en devient inopérant.

La faveur accordée sous réserve que l'entreprise ne détienne pas plus de $50 \%$ de patrimoine administratif ne trouve sa source dans aucune justification légale valable. En outre, le principe du « tout ou rien » conduit à une rupture d'égalité avec des entreprises ayant un patrimoine administratif légèrement supérieur à $50 \%$. La loi est à l'origine d'une incitation fâcheuse visant à échapper systématiquement à cette limite de 50 \% grâce à une libre identification des actifs.

La loi autorise certaines restructurations qui ne sont ni visées par la loi, ni justifiées en termes d'égalité. Parmi celles-ci, il faut en particulier citer les scissions d'entreprises (Betriebsaufspaltung) conduites en vue d'éviter la contrainte de la conservation de la masse salariale, ainsi que la réorganisation et la répartition du patrimoine administratif dans les groupes (effet de cascade).

La Cour constitutionnelle a décidé que le législateur devait produire une nouvelle réglementation conforme à la Constitution d'ici le 30 juin 2016.

\section{La réforme en débat}

\section{Le projet de loi du gouvernement fédéral du 7 juin 2015}

Au mois de juin 2015, le gouvernement a présenté un projet de loi qui fait suite à la liste de manquements constatés par le Tribunal constitutionnel fédéral. II a cependant opté pour une intervention a minima. Ainsi, l'exonération légale et l'exonération sur option sont maintenues. La règle de la masse salariale est quant à elle renforcée, et les durées de conservation de l'entreprise de cinq à sept ans sont reconduites.

Les actifs objets du régime de faveur sont comme avant les biens agricoles et forestiers, les actifs d'une entreprise, les actions de sociétés de capitaux correspondant à une détention supérieure à $25 \%$ et les participations dans des so-
Conservation de l'entreprise

Constitutionnalité de l'exonération légale, mais...

... tenir compte des besoins réels des entreprises...

... et du nombre de salariés,...

... revoir le seuil de $\mathbf{5 0} \%$ du patrimoine administratif...

... et éviter les effets de cascade

Refonte de la définition des actifs ouvrant droit au régime de faveur... 
... selon cinq étapes : ...

1. distinguer les actifs...

2. répartir les moyens financiers...

3. et les dettes...

4. réviser certains seuils...

5. et le calcul du taux du régime de faveur

Base de calcul pour les groupes : bilan consolidé

Exonération réduite pour les grosses entreprises ciétés de personnes. A ce propos, les participations dans des sociétés de personnes ayant une empreinte commerciale (gewerblich geprägt) ne peuvent faire l'objet du régime de faveur que dans la mesure où elles concernent des sociétés pouvant faire elles-mêmes l'objet d'une telle faveur. II en va de même pour les sociétés holdings, qui ne peuvent bénéficier du régime de faveur que si elles détiennent des sociétés qui peuvent également en bénéficier.

Le projet prévoit qu'il convient de déterminer les actifs faisant l'objet du régime de faveur selon cinq étapes :

Les actifs sont à classer respectivement entre ceux faisant l'objet du régime de faveur et ceux qui n'en font pas partie. Les actifs qui font l'objet du régime de faveur sont ceux qui servent principalement un but agricole ou forestier ou une profession indépendante ou un but commercial.

Dans un deuxième temps, il convient de répartir les moyens financiers, lesquels, comme précédemment, font partie des actifs appartenant au régime de faveur à hauteur de $20 \%$ de la valeur totale des actifs d'une entreprise. Sont à exonérer les moyens financiers «jeunes », qui ont été introduits pendant les deux dernières années dans l'actif de l'entreprise.

La troisième étape consiste à attribuer les dettes respectivement aux actifs ouvrant droit au régime de faveur et à ceux n'ouvrant pas droit à un tel régime. Cette allocation est toutefois permise non pas en fonction du lien avec l'actif qu'elles financent, mais en fonction du rapport entre la valeur de l'actif ouvrant droit au régime de faveur et celle de l'actif n'ouvrant pas droit à ce régime.

Lorsque l'actif n'ouvrant pas droit au régime de faveur atteint seulement $10 \%$ de la valeur de l'actif net, il ne peut alors pas être considéré comme tel et est traité comme un actif permettant l'application du régime de faveur.

Le taux du régime de faveur se détermine d'après le rapport entre la valeur nette de l'actif ouvrant droit au régime et la valeur nette de l'actif d'ensemble.

En raison de l'effet de cascade constaté dans les groupes, critique relevée par la Cour fédérale des finances, une présentation d'ensemble de l'actif doit être établie. Tous les actifs de l'entreprise et des sociétés situés en-dessous doivent être reportés dans un bilan consolidé établissant la valeur de l'actif du groupe. Ainsi, les transferts de moyens financiers qui peuvent avoir lieu au sein du groupe deviennent sans effet. Cet exercice constitue un réel défi pour les groupes car chaque actif doit être valorisé puis classé. II faut inclure dans la présentation d'ensemble de l'actif les participations dans des sociétés de personnes, les actions dans les sociétés de capitaux correspondant à une participation supérieure à $25 \%$ ainsi que les actions de sociétés de capitaux correspondant à une participation inférieure à $25 \%$ lorsqu'elle sert l'objet principal de la société.

En principe, il n'existe pas de régime de faveur pour l'acquisition à titre gratuit d'actifs, pouvant ouvrir droit à l'exonération, d'une valeur supérieure à 26 millions $€$. II est toutefois possible d'appliquer une exonération réduite de $20 \%$ maximum (respectivement $35 \%$ sur option) pour des acquisitions supérieures à 116 millions $€$. Une autre option est également ouverte à l'héritier ou donataire dans le cadre d'une acquisition d'une grosse entreprise : celui-ci peut solliciter un examen individualisé de sa situation personnelle en vue de bénéficier d'une exonération (voir ci-dessous).

L'exonération réduite est octroyée sur la base d'une demande irrévocable et fonctionne comme suit : l'exonération de $85 \%$ dans le cadre de l'exonération légale et celle de $100 \%$ sur option sont réduites de $1 \%$ pour chaque 1,5 million $€$ au-delà du seuil de 26 millions $€$. A partir d'une acquisition à titre gratuit d'actifs ouvrant droit au régime de faveur d'une valeur de 116 millions $€$, il n'existe plus d'exonération supplémentaire. L'exonération maximale est alors de $20 \%$, ou de $35 \%$ sur option. 
En outre, les règles existantes relatives à la masse salariale et aux durées de conservation doivent être remplies.

Le seuil de 26 millions $€$ est porté à 52 millions $€$, et le plafond de 116 millions $€$ passe à 142 millions $€$, mais dans des cas spécifiques, supposant des engagements contraignants et pouvant aller jusqu'à une durée de 30 ans après transmission.

Une alternative à l'exonération réduite peut être trouvée dans l'exonération des droits de successions si l'acquéreur prouve qu'il est personnellement dans l'incapacité d'acquitter les droits avec son patrimoine disponible. Cela signifie qu'il doit révéler l'intégralité de sa situation patrimoniale.

Le patrimoine disponible correspond à $50 \%$ de la somme des valeurs marchandes

- du patrimoine transmis par la succession ou la donation, qui ne fait pas partie du patrimoine ouvrant droit au régime de faveur, et

- du patrimoine de l'acquéreur au moment de l'acquisition, qui ne fait pas partie du patrimoine ouvrant droit au régime de faveur.

Il est possible de s'interroger sur la volonté d'un héritier/donataire de révéler son entier patrimoine auprès de l'administration fiscale afin de bénéficier d'une potentielle exonération fiscale. Le texte du projet de loi prévoit encore de façon alternative des possibilités de sursis de paiement sur une plus courte période.

L'exonération est soumise à la condition résolutoire que soient respectés

- le délai de conservation de sept ans et

- la masse salariale pendant sept ans.

Le projet de loi a pris acte de la critique relevée par la Cour de Karlsruhe et adapté les règles sur la masse salariale.

Comme précédemment, les entreprises ayant jusqu'à trois salariés ne sont pas dans l'obligation de maintenir la masse salariale.

Pour les entreprises ayant de quatre à dix salariés, $250 \%$ de la masse salariale sont à conserver pendant cinq ans dans le cadre de l'exonération légale, et $500 \%$ de la masse salariale sont à conserver pendant sept ans dans le cadre de l'exonération sur option.

Pour les entreprises ayant entre onze et quinze salariés, $300 \%$ de la masse salariale sont à conserver pendant plus de cinq ans dans le cadre de l'exonération légale, et $575 \%$ dans le cadre de l'exonération sur option pendant plus de sept ans.

Les entreprises ayant plus de quinze salariés doivent désormais respecter les règles relatives à la conservation de la masse salariale. Cela signifie que, s'agissant de l'exonération légale, $400 \%$ de la masse salariale de départ sont à conserver pendant cinq ans et, s'agissant de l'exonération sur option, $700 \%$ de la masse salariale de départ sont à conserver pendant sept ans.

En cas de scission de l'entreprise, le montant total des salaires, et donc le nombre de salariés, sont à compter ensemble.

\section{La critique du Bundesrat : avis du 25 septembre 2015}

Le 25 septembre 2015, le Bundesrat (i.e. la représentation des Länder) a pris position sur le projet de loi portant sur la loi fiscale sur les successions et donations. En effet, dans l'organisation du fédéralisme financier allemand, Bund, Länder et communes se répartissent l'encours des différents impôts. L'impôt sur les successions, dont le produit revient aux Länder, relève des compétences législatives concurrentes Bund/Länder qui attribue à la Fédération le droit de légiférer notamment lorsque «la sauvegarde de l'unité juridique ou économique dans l'intérêt de l'ensemble de l'Etat rendent nécessaire une réglementation législative fédérale » (art. 72 al. 2 de la Loi fondamentale). L'adoption de la future loi exige donc un compromis entre le Bund et les 16 Länder.
Seuil d'examen qualifié dans les entreprises familiales

Exonération dans le cas d'un examen de la situation patrimoniale personnelle

Changement de la masse salariale 
Catalogue du patrimoine administratif modifié

Exonération plancher

Règle du taux dégressif dans les cas de transmission

L'examen des besoins et la réduction du seuil d'examen
Le Bundesrat voit d'un œil critique le classement des actifs selon l'approche du but principal. Car pour chaque actif, il faudrait vérifier s'il pourrait être détaché de l'entreprise sans porter atteinte à l'activité commerciale. Le Bundesrat redoute ici une forte propension aux litiges et une gestion complexe tant pour les entreprises que pour l'administration fiscale. C'est pourquoi le Bundesrat a plaidé pour l'attachement au catalogue du patrimoine administratif comme délimitation négative pour définir la notion d'actif ouvrant droit au régime de faveur.

La limite de l'exonération (20\% pour l'exonération légale, $35 \%$ pour l'exonération sur option pour les grosses acquisitions) s'applique sans examen du besoin de l'exonération. Elle devrait donc être supprimée selon le Bundesrat.

Dans le cas des acquisitions supérieures à 26 millions $€$ (s'agissant des entreprises familiales: 52 millions $€$ ), la règle de réduction progressive du taux d'exonération s'applique. Pour les acquisitions supérieures à 116 millions $€$ (142 millions $€$ pour les entreprises familiales) elle prend fin : le taux devient unique, fixé à $20 \%$ ou $35 \%$ sur option. Le Bundesrat estime que la nouvelle règle n'apporterait pas de réponse satisfaisante à la critique de la Cour de Karlsruhe en ce sens qu'elle ne prend pas en compte les besoins spécifiques des entreprises concernées. Le Bundesrat estime par ailleurs que les seuils proposés doivent être sensiblement réduits.

Le Bundesrat critique le seuil de 26 millions $€$ par acquisition à titre gratuit (52 millions $€$ par acquisition s'agissant des entreprises familiales) car en 2013, seulement $1,69 \%$ des acquisitions d'actifs d'entreprises supérieurs à 20 millions $€$ a été transmis dans l'ensemble de l'Allemagne, ce qui veut dire qu'une grande partie des acquéreurs entraient dans le champ de l'exonération légale sans avoir à solliciter un examen des besoins de l'exonération. C'est pourquoi le Bundesrat estime impératif d'abaisser le seuil d'examen.

\section{Perspective et calendrier}

A l'origine, il était prévu de promulguer cette adaptation législative cette année. Suite à l'audience publique de la commission des finances du Bundestag, la promulgation initialement prévue en novembre a été reportée à l'année prochaine. En effet, le gouvernement fédéral et le Bundesrat entretiennent des positions divergentes en matière de politique fiscale, propres à ralentir le processus législatif. Les négociations sont d'autant plus délicates et les positions d'autant plus tranchées que la réforme du droit des successions intervient au moment où se négocie parallèlement une réforme de fond des règles de fonctionnement du fédéralisme financier, autrement dit de la question de la répartition de l'encours des différents types d'impôts revenant aux différents échelons territoriaux.

Il existe également, dans les milieux spécialisés, des partisans d'un taux unique plus bas dans l'hypothèse d'un élargissement de l'assiette de l'impôt. Pour l'instant, l'incertitude quant à la forme définitive du projet de loi et son entrée en vigueur demeure entière.

En outre, d'autres voix se font entendre en vue d'obtenir la notification auprès de la Commission européenne du régime de faveur accordé aux entreprises familiales comme étant une aide d'Etat conforme au droit de l'Union européenne.

SOUPLESSE DE LA TRANSMISSION D'ENTREPRISE OU RIGUEUR DE LA PROTECTION de l'entreprise ? La difficulté de parvenir à une entente commune entre le Bundesrat et le gouvernement fédéral est évidente face à la multitude des enjeux poursuivis, et notamment ceux visant tantôt à faciliter la transmission d'entreprise, tantôt à protéger les salariés, l'entreprise, et finalement le système économique allemand tout entier. Au cœur du Mittelstand, il faut en effet avoir à l'esprit que la plupart des entreprises familiales allemandes sont des petites et moyennes entreprises et que près de 500 grandes entreprises familiales représentent 
$11 \%$ du chiffre d'affaires total des sociétés allemandes. S'il est vrai que des entreprises familiales sont également cotées en bourse, la propriété reste la valeur essentielle de l'entrepreneuriat. Par ailleurs, il semblerait que plus de 25000 entreprises organisent leur succession dans les années à venir. 400000 emplois seraient donc en jeu. Ainsi, en l'état actuel du projet, $66 \%$ des entreprises familiales estiment qu'elles devront diminuer leurs investissements, $52 \%$ pensent devoir supprimer des emplois. Au vu de ces chiffres, prudence et doigté sont de rigueur.

Tandis qu'une partie du SPD estime le projet de loi encore trop conciliant à l'égard des grandes entreprises familiales aux noms internationalement célèbres, et donc contraire à la 'justice fiscale', la CDU et la CSU voient ce projet comme étant encore une fois en deçà des possibilités légales existantes et n'entendent guère le laisser en l'état. Face à cet ensemble de facteurs, la conciliation des objectifs, très largement politiques, apparaît sensible, voire compromise. Ces débats s'inscrivent en effet dans le cadre plus large de la délicate réforme de fond des règles de fonctionnement du fédéralisme financier (voir $R E A$ 115/2014) qui doit être achevée en 2019. Dans le cadre de cette révision imposée par la Cour de Karlsruhe et qui concerne tant les mécanismes horizontaux de solidarité entre les Länder (Länderfinanzausgleich) que, plus généralement, les modes de répartition verticaux entre Bund, Länder et communes, de quelque $70 \%$ du produit total des différents impôts, les enjeux politiques, budgétaires et partisans s'entrelacent à l'extrême. Les négociations très tendues sur ces questions budgétaires donnent lieu à d'intenses tractations où chaque dossier, isolé de son contexte, sert de monnaie d'échange. C'est le cas de la réforme de l'impôt sur les successions dont le produit revient en propre aux Länder. Elle renvoie aussi à celle, toujours repoussée jusqu'ici, de l'IS et de l'IR...

\section{Indications bibliographiques}

- BACH S., «Erbschaftsteuer auf den Bund übertragen, Länder stärker an Umsatz- und Einkommenssteuer beteiligen ", dossier spécial Erbschaftsteuer, DIW Wochenbericht, $n^{\circ}$ 23/2015

- BACH S., MUDRACK T., «Erbschaftsteuer: Firmenprivilegien begrenzen, Steuerbelastung strecken », dossier spécial Erbschaftsteuer, DIW Wochenbericht, $\mathrm{n}^{\circ} 7 / 2015$

- BouRGEOIS I., "Vers une réforme de fond du fédéralisme financier ", Regards sur l'économie allemande, $\mathrm{n}^{\circ} 115$, hiver 2014

- BUNDESRAT, Drucksache 353/1/2015; www.bundesrat.de/SharedDocs/beratungsvorgaenge/201 5/0301-0400/0353-15.html?cms templateQueryString=Erbschaftsteuer\&cms fromSearch=true

- BUNDESTAG, Drucksache 18/5923; http://dip21.bundestag.de/dip21/btd/18/059/1805923.pdf

- BUNDESVERFASSUNGSGERICHT, arrêt du 17 décembre 2014, n 1 BvL 21/12; www.bundesverfass ungsgericht.de/SharedDocs/Entscheidungen/DE/2014/12/ss20141217_1bvl002112.html. Communiqué de presse en anglais : www.bundesverfassungsgericht.de/Sharē̄Docs/Pressemitteilungen/EN/ 2014/bvg14-116.html

- Erbschaftssteuer- und Schenkungssteuergesetz (ErbStG), www.gesetze-im-internet.de/bundesrech t/erbstg_1974/gesamt.pdf

- Positions de la Chambre des experts fiscaux : Bundessteuerberaterkammer ; www.bstbk.de/de/ presse/stellungnahmen/archiv/20150908_stellungnahme_bstbk/index.html.

- Positions de la Fondation des entreprises familiales allemandes : www.familienunternehmen.de/ Erbschaftsteuer------_site.site..Is_dir._nav.261_likecms.html 